\title{
A note on rate of convergence of double singular integral operators
}

\author{
Mine Menekse Yilmaz ${ }^{1 *}$, Gumrah Uysal ${ }^{2}$ and Ertan $\mid$ bikli ${ }^{3}$
}

"Correspondence:

menekse@gantep.edu.tr

'Department of Mathematics,

Faculty of Arts and Science,

Gaziantep University, Gaziantep, Turkey

Full list of author information is available at the end of the article

\begin{abstract}
In this paper we prove the pointwise convergence and the rate of pointwise convergence for a family of singular integral operators with radial kernel in two-dimensional setting in the following form: $L_{\lambda}(f ; x, y)=\iint_{D} f(s, t) H_{\lambda}(s-x, t-y) d s d t$, $(x, y) \in D$, where $D=\langle a, b\rangle \times\langle c, d\rangle(\langle a, b\rangle \times\langle c, d\rangle$ is an arbitrary closed, semi-closed or open region in $\mathbb{R}^{2}$ ) and $\lambda \in \Lambda, \Lambda$ is a set of non-negative numbers with accumulation point $\lambda_{0}$. Also we provide an example to support these theoretical results.
\end{abstract}

MSC: Primary 41A35; secondary 41A25

Keywords: $p$-generalized Lebesgue point; radial kernel; pointwise convergence; rate of convergence

\section{Introduction}

Taberski [1] studied the pointwise convergence of integrable functions and the approximation properties of derivatives of integrable functions in $L_{1}(-\pi, \pi)$ by a family of convolution type singular integral operators depending on two parameters of the form:

$$
U_{\lambda}(f ; x)=\int_{-\pi}^{\pi} f(t) K_{\lambda}(t-x) d t, \quad x \in(-\pi, \pi)
$$

where $K_{\lambda}(t)$ is the kernel satisfying suitable assumptions and $\lambda \in \Lambda$ (where $\Lambda$ is a given set of non-negative numbers with accumulation point $\lambda_{0}$ ).

Based on Taberski's study [1], Gadjiev [2] investigated both the pointwise convergence theorems and the order of pointwise convergence theorems for operators type (1) at a generalized Lebesgue point. Then Rydzewska [3] conducted a similar study by changing the point to a $\mu$-generalized Lebesgue point of $f \in L_{1}(-\pi, \pi)$ instead of a generalized Lebesgue point.

Further, in [4, 5], Karsli and Ibikli extended the results of [1, 2], and [3] by considering the more general integral operators defined by

$$
T_{\lambda}(f ; x)=\int_{a}^{b} f(t) K_{\lambda}(t-x) d t, \quad x \in\langle a, b\rangle, \lambda \in \Lambda \subset \mathbb{R}
$$

for functions in $L_{1}\langle a, b\rangle$ where $\langle a, b\rangle$ is an arbitrary interval in $\mathbb{R}$ such as $[a, b],(a, b),[a, b)$ or $(a, b]$.

@2014 Yilmaz et al.; licensee Springer. This is an Open Access article distributed under the terms of the Creative Commons Attribution License (http://creativecommons.org/licenses/by/2.0), which permits unrestricted use, distribution, and reproduction in any medium, provided the original work is properly cited. 
In [6, 7], Karsli obtained the pointwise convergence theorems and the rate of pointwise convergence theorems for a family of nonlinear singular integral operators at a $\mu$-generalized Lebesgue point and a generalized Lebesgue point of $f \in L_{1}\langle a, b\rangle$, respectively.

In 1984, Bardaro and Gori Cocchieri [8] estimated the degree of pointwise convergence of Fejer-Type singular integrals at the generalized Lebesgue points of the functions $f \in L_{1}(\mathbb{R})$. Also, Bardaro [9] studied similar convergence results concerning moment type operators.

In [10], Bardaro and Mantellini investigated the pointwise convergence of family of nonlinear Mellin type convolution operators at Lebesgue points.

In paper [11], Bardaro et al. obtained some approximation results concerning the pointwise convergence and the rate of pointwise convergence for non-convolution type linear operators at a Lebesgue point. In [12], the same authors also obtained similar results for its nonlinear counterpart and then in [13], they explored the pointwise convergence and the rate of pointwise convergence results for a family of Mellin type nonlinear $m$-singular integral operators at $m$-Lebesgue points of $f$.

In this study, we also investigated the pointwise convergence and the rate of convergence of the operators similar to the studies above. However, this study considers the twodimensional singular integral instead of one-dimensional integral similar to what Taberski [14] investigated in 1964. In [14], Taberski explored the pointwise convergence of integrable functions in $L_{1}(Q)$ by a three-parameter family of convolution type singular integral operators of the form

$$
U_{\lambda}(f ; x, y)=\iint_{Q} f(s, t) K_{\lambda}(s-x, t-y) d s d t, \quad(x, y) \in Q,
$$

where $Q$ denotes a given rectangle. Based on Taberski's study [14], Siudut [15, 16] obtained significant results relating the pointwise convergence of singular integrals by considering the operators of type (3).

In [17] Yilmaz et al. studied the pointwise convergence of the singular integral operators in the following form:

$$
L_{\lambda}(f ; x, y)=\int_{-\pi}^{\pi} \int_{-\pi}^{\pi} f(s, t) H_{\lambda}(s-x, t-y) d s d t, \quad(x, y) \in\langle-\pi, \pi\rangle \times\langle-\pi, \pi\rangle
$$

to the function $f\left(x_{0}, y_{0}\right)$ in the case $(x, y, \lambda) \rightarrow\left(x_{0}, y_{0}, \lambda_{0}\right)$ in $L_{1}(\langle-\pi, \pi\rangle \times\langle-\pi, \pi\rangle)$, where $\langle-\pi, \pi\rangle \times\langle-\pi, \pi\rangle$ is a closed, semi-closed or open region in $\mathbb{R}^{2}$ and $\left(x_{0}, y_{0}\right)$ is a generalized Lebesgue point of the function $f \in L_{1}(\langle-\pi, \pi\rangle \times\langle-\pi, \pi\rangle)$. In this study, the kernel function $H_{\lambda}(s, t)$ is chosen as a radial function.

Very recently, [18] Serenbay et al. investigated the pointwise convergence of the operator of type (4) at a $\mu$-generalized Lebesgue point.

The purpose of this paper is to investigate the pointwise convergence and the rate of convergence of the operators in the following form:

$$
L_{\lambda}(f ; x, y)=\iint_{D} f(s, t) H_{\lambda}(s-x, t-y) d s d t, \quad(x, y) \in D,
$$

where $D=\langle a, b\rangle \times\langle c, d\rangle(\langle a, b\rangle \times\langle c, d\rangle$ is an arbitrary closed, semi-closed or open region in $\left.\mathbb{R}^{2}\right)$, at a $p$-generalized Lebesgue point of $f \in L_{p}(D)$ as $(x, y, \lambda) \rightarrow\left(x_{0}, y_{0}, \lambda_{0}\right)$. Here $L_{p}(D)$ 
is the collection of all measurable functions $f$ for which $|f|^{p}$ is integrable on $D(1 \leq p<\infty)$, $\Lambda$ is a set of non-negative numbers with accumulation point $\lambda_{0}$ and the kernel function $H_{\lambda}(s, t)$ is a radial function.

The paper is organized as follows: In Section 2, we introduce the fundamental definitions. In Section 3, we prove the existence of the operator of type (5). In Section 4, we obtain two theorems concerning the pointwise convergence of $L_{\lambda}(f ; x, y)$ to $f\left(x_{0}, y_{0}\right)$ whenever $\left(x_{0}, y_{0}\right)$ is a $p$-generalized Lebesgue point of $f$ in bounded region and unbounded region. In Section 5, we establish the rate of convergence of operators of type (5) to $f\left(x_{0}, y_{0}\right)$ as $(x, y, \lambda)$ tends to $\left(x_{0}, y_{0}, \lambda_{0}\right)$ and we conclude the paper with an example to support our results.

\section{Preliminaries}

In this section we introduce the main definitions used in this paper.

Definition 2.1 Let $\varphi(x, y)$ be a function defined in the rectangle $D$, let

$$
P=\left\{\begin{array}{l}
a=x_{1}<x_{2}<\cdots<x_{i}<\cdots<x_{m}<x_{m+1}=b, \\
c=y_{1}<y_{2}<\cdots<y_{j}<\cdots<y_{n}<y_{n+1}=d
\end{array}\right.
$$

be a partition of $D$ and

$$
\Delta \varphi\left(x_{i}, y_{j}\right)=\varphi\left(x_{i}, y_{j}\right)-\varphi\left(x_{i+1}, y_{j}\right)-\varphi\left(x_{i}, y_{j+1}\right)+\varphi\left(x_{i+1}, y_{j+1}\right)
$$

If $\Delta \varphi\left(x_{i}, y_{j}\right) \geq 0(i=1,2, \ldots, m ; j=1,2, \ldots, n)$ for any partition of $P$, then it is said that $\varphi(x, y)$ satisfies the condition $\Omega$ in $D$ [14].

In other words, if $\Delta \varphi\left(x_{i}, y_{j}\right) \geq 0$ for all partitions of $D$ then it is said that $\varphi(x, y)$ is bimonotonically increasing and if $\Delta \varphi\left(x_{i}, y_{j}\right) \leq 0$ for all partitions of $D$, then it is said that $\varphi(x, y)$ is bimonotonically decreasing [19].

Definition 2.2 A point $\left(x_{0}, y_{0}\right) \in D$ is called a $p$-generalized Lebesgue point of function $f \in L_{p}(D)$ if

$$
\begin{aligned}
& \lim _{(h, k) \rightarrow(0,0)}\left(\frac{1}{h^{\alpha+1} k^{\alpha+1}} \int_{0}^{h} \int_{0}^{k}\left|f\left(s+x_{0}, t+y_{0}\right)-f\left(x_{0}, y_{0}\right)\right|^{p} d s d t\right)^{\frac{1}{p}}=0 \\
& (0 \leq \alpha<1 ; 1 \leq p<\infty) .
\end{aligned}
$$

Definition 2.3 A function $H \in L_{1}\left(\mathbb{R}^{2}\right)$, is said to be radial if there exists a function $K$ : $\mathbb{R}_{0}^{+} \rightarrow \mathbb{R}$ such that $H(s, t)=K\left(\sqrt{s^{2}+t^{2}}\right)$ a.e. [20].

Example 2.1 Let $H: \mathbb{R}^{2} \rightarrow \mathbb{R}$ is given by $H(s, t)=e^{-\left(s^{2}+t^{2}\right)}$ and the corresponding function $K: \mathbb{R}_{0}^{+} \rightarrow \mathbb{R}$ is $K(z)=e^{-z^{2}}$. Since the following equality holds for all $(s, t) \in \mathbb{R}^{2}$ :

$$
e^{-\left(s^{2}+t^{2}\right)}=e^{-\left(\sqrt{s^{2}+t^{2}}\right)^{2}},
$$

the function $H(s, t)$ is a radial function. 
Definition 2.4 (Class $A$ ) Let $H_{\lambda}: \mathbb{R}^{2} \times \Lambda \rightarrow \mathbb{R}$ be a radial function i.e., there exists a function $K_{\lambda}: \mathbb{R}_{0}^{+} \times \Lambda \rightarrow \mathbb{R}$ such that the following equality holds for $(s, t) \in \mathbb{R}^{2}$ a.e.

$$
H_{\lambda}(s, t):=K_{\lambda}\left(\sqrt{s^{2}+t^{2}}\right),
$$

where $\Lambda$ is a given set of non-negative numbers with accumulation point $\lambda_{0}$.

We will say that the function $H_{\lambda}(s, t)$ belongs to class $A$, if the following conditions are satisfied:

(a) $H_{\lambda}(s, t)=K_{\lambda}\left(\sqrt{s^{2}+t^{2}}\right)$ is non-negative and integrable as a function of $(s, t)$ on $\mathbb{R}^{2}$ for each fixed $\lambda \in \Lambda$.

(b) For fixed $\left(x_{0}, y_{0}\right) \in D, K_{\lambda}\left(\sqrt{x_{0}^{2}+y_{0}^{2}}\right)$ tends to infinity as $\lambda$ tends to $\lambda_{0}$.

(c) $\lim _{(x, y, \lambda) \rightarrow\left(x_{0}, y_{0}, \lambda_{0}\right)} \iint_{\mathbb{R}^{2}} K_{\lambda}\left(\sqrt{(s-x)^{2}+(t-y)^{2}}\right) d s d t=1$.

(d) $\lim _{\lambda \rightarrow \lambda_{0}}\left[\sup _{\xi \leq \sqrt{s^{2}+t^{2}}} K_{\lambda}\left(\sqrt{s^{2}+t^{2}}\right)\right]=0, \forall \xi>0$.

(e) $\lim _{\lambda \rightarrow \lambda_{0}}\left[\iint_{\xi \leq \sqrt{s^{2}+t^{2}}} K_{\lambda}\left(\sqrt{s^{2}+t^{2}}\right) d s d t\right]=0, \forall \xi>0$.

(f) $K_{\lambda}\left(\sqrt{(s-x)^{2}+(t-y)^{2}}\right)$ is non-increasing with respect to $t$ on $\left\langle x_{0}, d\right\rangle$ and non-decreasing on $\left\langle c, x_{0}\right\rangle$ and similarly $K_{\lambda}\left(\sqrt{(s-x)^{2}+(t-y)^{2}}\right)$ is non-increasing with respect to $s$ on $\left\langle y_{0}, b\right\rangle$ and non-decreasing on $\left\langle a, y_{0}\right\rangle$, for any $\lambda \in \Lambda$ and for fixed $\left(x_{0}, y_{0}\right) \in D$.

(g) $\left\|K_{\lambda}\right\|_{L_{1}\left(\mathbb{R}^{2}\right)} \leq M, \forall \lambda \in \Lambda$.

Throughout this paper we suppose that the kernel $H_{\lambda}(s, t)$ belongs to class $A$.

\section{Existence of the operator}

Lemma 3.1 Let $1 \leq p<\infty$. If $f \in L_{p}(D)$, then the operator $L_{\lambda}(f ; x, y)$ defines a continuous transformation over $L_{p}(D)$.

Proof The proof of the case $p=1$ is quite similar to the proof in [21].

We assume that $1<p<\infty$. By the linearity of the operator $L_{\lambda}(f ; x, y)$, it is sufficient to show that the following expression is bounded:

$$
\left\|L_{\lambda}\right\|=\sup _{f \neq 0} \frac{\left\|L_{\lambda}(f, x, y)\right\|_{L_{p}(D)}}{\|f\|_{L_{p}(D)}} .
$$

We define a function such that

$$
g(s, t)= \begin{cases}f(s, t), & (s, t) \in D, \\ 0, & (s, t) \in \mathbb{R}^{2} \backslash D,\end{cases}
$$

and then we rearrange and rewrite the norm as follows:

$$
\begin{aligned}
\left\|L_{\lambda}(f, x, y)\right\|_{L_{p}(D)} & =\left\|L_{\lambda}(g, x, y)\right\|_{L_{p}(D)} \\
& =\left(\iint_{D}\left|\iint_{\mathbb{R}^{2}} g(s, t) H_{\lambda}(s-x, t-y) d s d t\right|^{p} d x d y\right)^{\frac{1}{p}} \\
& =\left(\iint_{D}\left|\iint_{\mathbb{R}^{2}} g(s, t) K_{\lambda}\left(\sqrt{(s-x)^{2}+(t-y)^{2}}\right) d s d t\right|^{p} d x d y\right)^{\frac{1}{p}} \\
& =\left(\iint_{D}\left|\iint_{\mathbb{R}^{2}} g(s+x, t+y) K_{\lambda}\left(\sqrt{s^{2}+t^{2}}\right) d s d t\right|^{p} d x d y\right)^{\frac{1}{p}} .
\end{aligned}
$$


By using the generalized Minkowsky inequality and by condition (g) of class $A$, we have

$$
\begin{aligned}
\left\|L_{\lambda}(f, x, y)\right\|_{L_{p}(D)} & \leq \iint_{\mathbb{R}^{2}}\left(\iint_{D}|g(s+x, t+y)|^{p}\left|K_{\lambda}\left(\sqrt{s^{2}+t^{2}}\right)\right|^{p} d x d y\right)^{\frac{1}{p}} d s d t \\
& =\iint_{\mathbb{R}^{2}}\left|K_{\lambda}\left(\sqrt{s^{2}+t^{2}}\right)\right|\left(\iint_{D}|g(s+x, t+y)|^{p} d x d y\right)^{\frac{1}{p}} d s d t \\
& =\iint_{\mathbb{R}^{2}}\left|K_{\lambda}\left(\sqrt{s^{2}+t^{2}}\right)\right|\left(\int_{a+s}^{b+s} \int_{c+t}^{d+t}|g(u, v)|^{p} d u d v\right)^{\frac{1}{p}} d s d t \\
& \leq \iint_{\mathbb{R}^{2}}\left|K_{\lambda}\left(\sqrt{s^{2}+t^{2}}\right)\right|\left(\int_{a}^{b} \int_{c}^{d}|f(u, v)|^{p} d u d v\right)^{\frac{1}{p}} d s d t \leq M\|f\|_{L_{p}(D) .}
\end{aligned}
$$

Thus the proof is completed.

\section{Pointwise convergence}

The following theorem gives a pointwise approximation of the integral operators type (5) to the function $f$ at $p$-generalized Lebesgue point of $f \in L_{p}(D)$ whenever $D$ is an arbitrary region in $\mathbb{R}^{2}$ that is bounded, closed, semi-closed or open.

Theorem 4.1 Suppose that, as a function of $(s, t), \Delta K\left(\sqrt{(t-x)^{2}+(s-y)^{2}} ; \lambda\right) \geq 0$ on $\left\langle x_{0}, b\right\rangle \times\left\langle y_{0}, d\right\rangle$ and $\left\langle a, x_{0}\right\rangle \times\left\langle c, y_{0}\right\rangle, \Delta K\left(\sqrt{(t-x)^{2}+(s-y)^{2}} ; \lambda\right) \leq 0$ on $\left\langle x_{0}, b\right\rangle \times\left\langle c, y_{0}\right\rangle$ and $\left\langle a, x_{0}\right\rangle \times\left\langle y_{0}, d\right\rangle$.If $\left(x_{0}, y_{0}\right)$ is a p-generalized Lebesgue point of function $f \in L_{p}(D)$, then

$$
\lim _{(x, y, \lambda) \rightarrow\left(x_{0}, y_{0}, \lambda_{0}\right)} L_{\lambda}(f ; x, y)=f\left(x_{0}, y_{0}\right)
$$

on any set $Z$ on which the function

$$
\int_{x_{0}-\delta}^{x_{0}+\delta} \int_{y_{0}-\delta}^{y_{0}+\delta} K_{\lambda}\left(\sqrt{(s-x)^{2}+(t-y)^{2}}\right)\left|d\left[\left(s-x_{0}\right)^{p(\alpha+1)}\left(t-y_{0}\right)^{p(\alpha+1)}\right]\right|,
$$

where $d\left[\left(x_{0}-s\right)^{p(\alpha+1)}\left(y_{0}-t\right)^{p(\alpha+1)}\right]$ is the Lebesgue-Stieltjes measure with respect to $\left(x_{0}-\right.$ $s)^{p(\alpha+1)}\left(y_{0}-t\right)^{p(\alpha+1)}$, is bounded as $(x, y, \lambda)$ tends to $\left(x_{0}, y_{0}, \lambda_{0}\right)$.

Proof Suppose that $\left(x_{0}, y_{0}\right) \in D,\left|x_{0}-x\right|<\frac{\delta}{2}$, for all $\delta>0$ which satisfy $x_{0}+\delta<b$ and $x_{0}-\delta>$ $a,\left|y_{0}-y\right|<\frac{\delta}{2}$ for all $\delta>0$ which satisfy $y_{0}+\delta<d$ and $y_{0}-\delta>c$.

Since $\left(x_{0}, y_{0}\right) \in D$ is a $p$-generalized Lebesgue point of function $f \in L_{p}(D)$, for all given $\varepsilon>0$, there exists a $\delta>0$ such that for all $h, k$ satisfying $0<h, k \leq \delta$, the inequalities

$$
\begin{aligned}
& \int_{x_{0}-\delta}^{x_{0}} \int_{y_{0}-\delta}^{y_{0}}\left|f(s, t)-f\left(x_{0}, y_{0}\right)\right|^{p} d s d t<\varepsilon^{p}(h k)^{p(\alpha+1)}, \\
& \int_{x_{0}}^{x_{0}+\delta} \int_{y_{0}-\delta}^{y_{0}}\left|f(s, t)-f\left(x_{0}, y_{0}\right)\right|^{p} d s d t<\varepsilon^{p}(h k)^{p(\alpha+1)}, \\
& \int_{x_{0}-\delta}^{x_{0}} \int_{y_{0}}^{y_{0}+\delta}\left|f(s, t)-f\left(x_{0}, y_{0}\right)\right|^{p} d s d t<\varepsilon^{p}(h k)^{p(\alpha+1)}, \\
& \int_{x_{0}}^{x_{0}+\delta} \int_{y_{0}}^{y_{0}+\delta}\left|f(s, t)-f\left(x_{0}, y_{0}\right)\right|^{p} d s d t<\varepsilon^{p}(h k)^{p(\alpha+1)}
\end{aligned}
$$

hold. 
Set $I_{\lambda}(x, y):=\left|L_{\lambda}(f ; x, y)-f\left(x_{0}, y_{0}\right)\right|$. According to condition (c) of class $A$, we shall write

$$
\begin{aligned}
I_{\lambda}(x, y)= & \left|\iint_{D} f(s, t) H_{\lambda}(s-x, t-y) d s d t-f\left(x_{0}, y_{0}\right)\right| \\
= & \left|\iint_{D} f(s, t) K_{\lambda}\left(\sqrt{(s-x)^{2}+(t-y)^{2}}\right) d s d t-f\left(x_{0}, y_{0}\right)\right| \\
\leq & \iint_{\mathbb{R}^{2}}\left|g(s, t)-f\left(x_{0}, y_{0}\right)\right| K_{\lambda}\left(\sqrt{(s-x)^{2}+(t-y)^{2}}\right) d s d t \\
& +\left|f\left(x_{0}, y_{0}\right)\right|\left|\iint_{\mathbb{R}^{2}} K_{\lambda}\left(\sqrt{(s-x)^{2}+(t-y)^{2}}\right) d s d t-1\right| \\
= & \iint_{D}\left|f(s, t)-f\left(x_{0}, y_{0}\right)\right| K_{\lambda}\left(\sqrt{(s-x)^{2}+(t-y)^{2}}\right) d s d t \\
& +\left|f\left(x_{0}, y_{0}\right)\right|\left|\iint_{\mathbb{R}^{2}} K_{\lambda}\left(\sqrt{(s-x)^{2}+(t-y)^{2}}\right) d s d t-1\right| \\
& +\iint_{\mathbb{R}^{2} \backslash D}\left|g(s, t)-f\left(x_{0}, y_{0}\right)\right| K_{\lambda}\left(\sqrt{(s-x)^{2}+(t-y)^{2}}\right) d s d t \\
= & I+I I+I I I .
\end{aligned}
$$

By conditions (e) and (d) of class $A, I I I \rightarrow 0$ as $\lambda \rightarrow \lambda_{0}$.

Using Hölder's inequality for the term $I$ we have the following:

$$
\begin{aligned}
I+I I \leq & \left(\int_{a}^{b} \int_{c}^{d}\left|f(s, t)-f\left(x_{0}, y_{0}\right)\right|^{p}\left|K_{\lambda}\left(\sqrt{(s-x)^{2}+(t-y)^{2}}\right)\right| d s d t\right)^{\frac{1}{p}} \\
& \times\left(\int_{a}^{b} \int_{c}^{d} K_{\lambda}\left(\sqrt{(s-x)^{2}+(t-y)^{2}}\right) d s d t\right)^{\frac{1}{q}} \\
& +\left|f\left(x_{0}, y_{0}\right)\right|\left|\iint_{\mathbb{R}^{2}} K_{\lambda}\left(\sqrt{(s-x)^{2}+(t-y)^{2}}\right) d s d t-1\right|
\end{aligned}
$$

Since whenever for $m, n$ being positive numbers the inequality $(m+n)^{p} \leq 2^{p}\left(m^{p}+n^{p}\right)$ holds, by taking the $p$ th power of both sides we have

$$
\begin{aligned}
(I+I I)^{p} \leq & 2^{p}\left(\int_{a}^{b} \int_{c}^{d}\left|f(s, t)-f\left(x_{0}, y_{0}\right)\right|^{p} K_{\lambda}\left(\sqrt{(s-x)^{2}+(t-y)^{2}}\right) d s d t\right) \\
& \times\left(\iint_{\mathbb{R}^{2}} K_{\lambda}\left(\sqrt{(s-x)^{2}+(t-y)^{2}}\right) d s d t\right)^{\frac{P}{q}} \\
& +2^{p}\left|f\left(x_{0}, y_{0}\right)\right|^{p}\left|\iint_{\mathbb{R}^{2}} K_{\lambda}\left(\sqrt{(s-x)^{2}+(t-y)^{2}}\right) d s d t-1\right|^{p} \\
= & I^{*}+I I^{*} .
\end{aligned}
$$

Let us write

$$
\begin{aligned}
I^{*}= & 2^{p}\left(\int_{a}^{b} \int_{c}^{d}\left|f(s, t)-f\left(x_{0}, y_{0}\right)\right|^{p} K_{\lambda}\left(\sqrt{(s-x)^{2}+(t-y)^{2}}\right) d s d t\right) \\
& \times\left(\iint_{\mathbb{R}^{2}} K_{\lambda}\left(\sqrt{(s-x)^{2}+(t-y)^{2}}\right) d s d t\right)^{\frac{p}{q}} .
\end{aligned}
$$


The second part of the integral $I^{*}$ tends to one as $(x, y, \lambda)$ tends to $\left(x_{0}, y_{0}, \lambda_{0}\right)$ by condition (c). Then we investigate the first part of the integral $I^{*}$, denoted by $I_{1}$,

$$
I_{1}=\left\{\iint_{D \backslash B_{\delta}}+\iint_{B_{\delta}}\right\}\left|f(s, t)-f\left(x_{0}, y_{0}\right)\right|^{p} K_{\lambda}\left(\sqrt{(s-x)^{2}+(t-y)^{2}}\right) d s d t=I_{11}+I_{12},
$$

where $B_{\delta}:=\left\{(s, t):\left(s-x_{0}\right)^{2}+\left(t-y_{0}\right)^{2} \leq \delta^{2},\left(x_{0}, y_{0}\right) \in D\right\}$.

For the integral $I_{11}$ we have the following inequality:

$$
\begin{aligned}
I_{11} & =\iint_{D \backslash B_{\delta}}\left|f(s, t)-f\left(x_{0}, y_{0}\right)\right|^{p} K_{\lambda}\left(\sqrt{(s-x)^{2}+(t-y)^{2}}\right) d s d t \\
& \leq \iint_{D \backslash B_{\delta}}\left(|f(s, t)|+\left|f\left(x_{0}, y_{0}\right)\right|\right)^{p} K_{\lambda}\left(\sqrt{(s-x)^{2}+(t-y)^{2}}\right) d s d t \\
& \leq 2^{p} \iint_{D \backslash B_{\delta}}\left(|f(s, t)|^{p}+\left|f\left(x_{0}, y_{0}\right)\right|^{p}\right) K_{\lambda}\left(\sqrt{(s-x)^{2}+(t-y)^{2}}\right) d s d t \\
& \leq 2^{p} \sup _{\xi \leq \sqrt{s^{2}+t^{2}}} K_{\lambda}\left(\sqrt{s^{2}+t^{2}}\right)\left(\|f\|_{L_{p}(D)}^{p}+\left|f\left(x_{0}, y_{0}\right)\right|^{p}|b-a||d-c|\right) .
\end{aligned}
$$

Since $K_{\lambda}\left(\sqrt{s^{2}+t^{2}}\right)$ is decreasing on $D \backslash B_{\delta}$, the following relation holds:

$$
I_{11} \leq 2^{p} K_{\lambda}\left(\frac{(\sqrt{2}-1) \delta}{\sqrt{2}}\right)\left(\|f\|_{L_{p}(D)}^{p}+\left|f\left(x_{0}, y_{0}\right)\right|^{p}|b-a||d-c|\right)
$$

hence by condition (d) of class $A, I_{11} \rightarrow 0$ whenever $(x, y, \lambda) \rightarrow\left(x_{0}, y_{0}, \lambda_{0}\right)$.

Next we can show that the term $I_{12}$ tends to zero as $(x, y, \lambda) \rightarrow\left(x_{0}, y_{0}, \lambda_{0}\right)$ on $B_{\delta}$ :

$$
\begin{aligned}
I_{12}= & \iint_{B_{\delta}}\left|f(s, t)-f\left(x_{0}, y_{0}\right)\right| K_{\lambda}\left(\sqrt{(s-x)^{2}+(t-y)^{2}}\right) d s d t \\
\leq & \int_{x_{0}-\delta}^{x_{0}+\delta} \int_{y_{0}-\delta}^{y_{0}+\delta}\left|f(s, t)-f\left(x_{0}, y_{0}\right)\right| K_{\lambda}\left(\sqrt{(s-x)^{2}+(t-y)^{2}}\right) d s d t \\
\leq & \left\{\int_{x_{0}-\delta}^{x_{0}} \int_{y_{0}-\delta}^{y_{0}}+\int_{x_{0}}^{x_{0}+\delta} \int_{y_{0}-\delta}^{y_{0}}\right\}\left|f(s, t)-f\left(x_{0}, y_{0}\right)\right| K_{\lambda}\left(\sqrt{(s-x)^{2}+(t-y)^{2}}\right) d s d t \\
& +\left\{\int_{x_{0}-\delta}^{x_{0}} \int_{y_{0}}^{y_{0}+\delta}+\int_{x_{0}}^{x_{0}+\delta} \int_{y_{0}}^{y_{0}+\delta}\right\}\left|f(s, t)-f\left(x_{0}, y_{0}\right)\right| K_{\lambda}\left(\sqrt{(s-x)^{2}+(t-y)^{2}}\right) d s d t \\
= & I_{121}+I_{122}+I_{123}+I_{124} .
\end{aligned}
$$

Since

$$
I_{12} \leq I_{121}+I_{122}+I_{123}+I_{124}
$$

it is sufficient to show that the terms on the right hand side of the last inequality tends to zero as $(x, y, \lambda) \rightarrow\left(x_{0}, y_{0}, \lambda_{0}\right)$ on $Z$.

Let us consider first the integral $I_{121}$.

From (6), for every $\varepsilon>0$ there exists a $\delta>0$ such that

$$
\int_{x_{0}-h}^{x_{0}} \int_{y_{0}-k}^{y_{0}}\left|f(s, t)-f\left(x_{0}, y_{0}\right)\right|^{p} d s d t<\varepsilon^{p} h^{p(\alpha+1)} k^{p(\alpha+1)}
$$

for all $0<h, k \leq \delta$. 
Let us define a new function as

$$
F(s, t):=\int_{s}^{x_{0}} \int_{t}^{y_{0}}\left|f(u, v)-f\left(x_{0}, y_{0}\right)\right|^{p} d u d v .
$$

Then for all $s$ and $t$ satisfying $x_{0}-s \leq \delta$ and $y_{0}-t \leq \delta$ we have

$$
|F(s, t)| \leq \varepsilon^{p}\left(x_{0}-s\right)^{p(\alpha+1)}\left(y_{0}-t\right)^{p(\alpha+1)} .
$$

Hence we can evaluate the integral $I_{121}$.

From [14] (see Theorem 2.5, p.101), we can write the following:

$$
\begin{aligned}
I_{121} & =\int_{x_{0}-\delta}^{x_{0}} \int_{y_{0}-\delta}^{y_{0}}\left|f(s, t)-f\left(x_{0}, y_{0}\right)\right| K_{\lambda}\left(\sqrt{(s-x)^{2}+(t-y)^{2}}\right) d s d t \\
& =(S) \int_{x_{0}-\delta}^{x_{0}} \int_{y_{0}-\delta}^{y_{0}} K_{\lambda}\left(\sqrt{(s-x)^{2}+(t-y)^{2}}\right) d F(s, t),
\end{aligned}
$$

where $(S)$ denotes the Stieltjes integral.

Two-dimensional integration by parts (see Theorem 2.2, p.100 in [14]) gives us

$$
\begin{aligned}
\int_{x_{0}-\delta}^{x_{0}} & \int_{y_{0}-\delta}^{y_{0}} K_{\lambda}\left(\sqrt{(s-x)^{2}+(t-y)^{2}}\right) d F(s, t) \\
= & \int_{x_{0}-\delta}^{x_{0}} \int_{y_{0}-\delta}^{y_{0}} F(s, t) d K_{\lambda}\left(\sqrt{(s-x)^{2}+(t-y)^{2}}\right) \\
& +\int_{x_{0}-\delta}^{x_{0}} F\left(s, y_{0}-\delta\right) d K_{\lambda}\left(\sqrt{(s-x)^{2}+\left(y_{0}-y-\delta\right)^{2}}\right) \\
& +\int_{y_{0}-\delta}^{y_{0}} F\left(x_{0}-\delta, t\right) d K_{\lambda}\left(\sqrt{\left(x_{0}-x-\delta\right)^{2}+(t-y)^{2}}\right) \\
& +F\left(x_{0}-\delta, y_{0}-\delta\right) K_{\lambda}\left(\sqrt{\left(x_{0}-x-\delta\right)^{2}+\left(y_{0}-y-\delta\right)^{2}}\right) .
\end{aligned}
$$

From (6), we can write

$$
\begin{aligned}
I_{121} \leq & \varepsilon^{p} \int_{x_{0}-\delta}^{x_{0}} \int_{y_{0}-\delta}^{y_{0}}\left(x_{0}-s\right)^{p(\alpha+1)}\left(y_{0}-t\right)^{p(\alpha+1)}\left|d K_{\lambda}\left(\sqrt{(s-x)^{2}+(t-y)^{2}}\right)\right| \\
& +\varepsilon^{p} \delta^{p(\alpha+1)} \int_{x_{0}-\delta}^{x_{0}}\left(x_{0}-s\right)^{p(\alpha+1)}\left|d_{s} K_{\lambda}\left(\sqrt{(s-x)^{2}+\left(y_{0}-\delta-y\right)^{2}}\right)\right| \\
& +\varepsilon^{p} \delta^{p(\alpha+1)} \int_{y_{0}-\delta}^{y_{0}}\left(y_{0}-t\right)^{p(\alpha+1)}\left|d_{t} K_{\lambda}\left(\sqrt{\left(x_{0}-\delta-x\right)^{2}+(t-y)^{2}}\right)\right| \\
& +\varepsilon^{p} \delta^{2 p(\alpha+1)} K_{\lambda}\left(\sqrt{\left(x_{0}-\delta-x\right)^{2}+\left(y_{0}-\delta-y\right)^{2}}\right) \\
= & i_{1}+i_{2}+i_{3}+i_{4} .
\end{aligned}
$$

After applying integration by parts to $i_{1}, i_{2}$, and $i_{3}$ we obtain the following result:

$$
I_{121} \leq \varepsilon^{p} \int_{x_{0}-\delta}^{x_{0}} \int_{y_{0}-\delta}^{y_{0}} K_{\lambda}\left(\sqrt{(s-x)^{2}+(t-y)^{2}}\right) d\left(x_{0}-s\right)^{p(\alpha+1)}\left(y_{0}-t\right)^{p(\alpha+1)} .
$$


For the integrals $I_{122}, I_{123}$, and $I_{124}$ the proof is similar to the above one. Thus we obtain the following inequalities:

$$
\begin{aligned}
& I_{122} \leq-\varepsilon^{p} \int_{x_{0}}^{x_{0}+\delta} \int_{y_{0}-\delta}^{y_{0}} K_{\lambda}\left(\sqrt{(s-x)^{2}+(t-y)^{2}}\right) d\left[\left(x_{0}-s\right)^{p(\alpha+1)}\left(y_{0}-t\right)^{p(\alpha+1)}\right], \\
& I_{123} \leq-\varepsilon^{p} \int_{x_{0}-\delta}^{x_{0}} \int_{y_{0}}^{y_{0}+\delta} K_{\lambda}\left(\sqrt{(s-x)^{2}+(t-y)^{2}}\right) d\left[\left(s-x_{0}\right)^{p(\alpha+1)}\left(y_{0}-t\right)^{p(\alpha+1)}\right], \\
& I_{124} \leq \varepsilon^{p} \int_{x_{0}}^{x_{0}+\delta} \int_{y_{0}}^{y_{0}+\delta} K_{\lambda}\left(\sqrt{(s-x)^{2}+(t-y)^{2}}\right) d\left[\left(x_{0}-s\right)^{p(\alpha+1)}\left(y_{0}-t\right)^{p(\alpha+1)}\right] .
\end{aligned}
$$

Collecting the estimates $I_{121}, I_{122}, I_{123}$, and $I_{124}$, we have the following inequality:

$$
\begin{aligned}
I_{12} & \leq I_{121}+I_{122}+I_{123}+I_{124} \\
& =\varepsilon^{p} \int_{x_{0}-\delta}^{x_{0}+\delta} \int_{y_{0}-\delta}^{y_{0}+\delta} K_{\lambda}\left(\sqrt{(s-x)^{2}+(t-y)^{2}}\right)\left|d\left[\left(x_{0}-s\right)^{p(\alpha+1)}\left(y_{0}-t\right)^{p(\alpha+1)}\right]\right| .
\end{aligned}
$$

Therefore, if the points $(x, y, \lambda) \in Z$ are sufficiently close to $\left(x_{0}, y_{0}, \lambda_{0}\right)$, we have

$$
I_{12} \leq \varepsilon C,
$$

where

$$
\begin{gathered}
C=\sup \left\{\int_{x_{0}-\delta}^{x_{0}+\delta} \int_{y_{0}-\delta}^{y_{0}+\delta} K_{\lambda}\left(\sqrt{(s-x)^{2}+(t-y)^{2}}\right)\left|d\left[\left(x_{0}-s\right)^{p(\alpha+1)}\left(y_{0}-t\right)^{p(\alpha+1)}\right]\right|:\right. \\
(x, y, \lambda) \in Z\} .
\end{gathered}
$$

Finally we consider the integral $I I^{*}$. From condition (c), it is clear that

$$
\lim _{(x, y, \lambda) \rightarrow\left(x_{0}, y_{0}, \lambda_{0}\right)} I I^{*}=0
$$

Thus, the proof is finished.

Remark 4.1 For the case $p=1$, the proof is quite similar to the proof in [21].

The following theorem gives a pointwise approximation of the integral operators type (5) to the function $f$ at $p$-generalized Lebesgue point of $f \in L_{p}\left(\mathbb{R}^{2}\right)$ whenever $D=\mathbb{R}^{2}$.

Theorem 4.2 Suppose that the hypothesis of Theorem 4.1 is satisfied for $D=\mathbb{R}^{2}$. If $\left(x_{0}, y_{0}\right)$ is a p-generalized Lebesgue point of function $f \in L_{p}\left(\mathbb{R}^{2}\right)$ then

$$
\lim _{(x, y, \lambda) \rightarrow\left(x_{0}, y_{0}, \lambda_{0}\right)} L_{\lambda}(f ; x, y)=f\left(x_{0}, y_{0}\right) .
$$

Proof Using the same strategy as in Theorem 4.1 we obtain

$$
\begin{aligned}
& \left|L_{\lambda}(f ; x, y)-f\left(x_{0}, y_{0}\right)\right|^{p} \\
& \quad \leq 2^{2 p}\left\{\sup _{\delta \leq \sqrt{s^{2}+t^{2}}}\left|K_{\lambda}\left(\sqrt{t^{2}+s^{2}}\right)\right|\|f\|_{L_{p}\left(\mathbb{R}^{2}\right)}^{p}\right.
\end{aligned}
$$




$$
\begin{aligned}
& \left.+\left|f\left(x_{0}, y_{0}\right)\right|^{p} \iint_{\delta \leq \sqrt{s^{2}+t^{2}}} K_{\lambda}\left(\sqrt{s^{2}+t^{2}}\right) d s d t\right\} \\
& \times\left(\iint_{\mathbb{R}^{2}} K_{\lambda}\left(\sqrt{(s-x)^{2}+(t-y)^{2}}\right) d s d t\right)^{\frac{p}{q}} \\
& +2^{p} \varepsilon^{p} \int_{x_{0}-\delta}^{x_{0}+\delta} \int_{y_{0}-\delta}^{y_{0}+\delta} K_{\lambda}\left(\sqrt{(s-x)^{2}+(t-y)^{2}}\right)\left|d\left[\left(x_{0}-s\right)^{p(\alpha+1)}\left(y_{0}-t\right)^{p(\alpha+1)}\right]\right| \\
& \times\left(\iint_{\mathbb{R}^{2}} K_{\lambda}\left(\sqrt{(s-x)^{2}+(t-y)^{2}}\right) d s d t\right)^{\frac{p}{q}} \\
& +2^{p}\left|f\left(x_{0}, y_{0}\right)\right|^{p}\left|\iint_{\mathbb{R}^{2}} K_{\lambda}\left(\sqrt{(s-x)^{2}+(t-y)^{2}}\right) d s d t-1\right|^{p} .
\end{aligned}
$$

By condition (e) of class $A$ the second term of the expression tends to zero as $\lambda$ tends to $\lambda_{0}$. The remaining part of the proof is obvious by Theorem 4.1 .

\section{Rate of convergence}

In this section, we give a theorem concerning the rate of pointwise convergence.

Theorem 5.1 Suppose that the hypotheses of Theorem 4.1 and Theorem 4.2 are satisfied.

Let

$$
\Delta(\lambda, \delta, x, y)=\int_{x_{0}-\delta}^{x_{0}+\delta} \int_{y_{0}-\delta}^{y_{0}+\delta}\left|x_{0}-s\right|^{p(\alpha+1)-1}\left|y_{0}-t\right|^{p(\alpha+1)-1} K_{\lambda}\left(\sqrt{(s-x)^{2}+(t-y)^{2}}\right) d s d t
$$

for $\delta>0$ and the following assumptions are satisfied:

(i) $\Delta(\lambda, \delta, x, y) \rightarrow 0$ as $(x, y, \lambda) \rightarrow\left(x_{0}, y_{0}, \lambda_{0}\right)$ for some $\delta>0$.

(ii) For every $\xi>0$

$$
\begin{aligned}
K_{\lambda}(\xi) & =o(\Delta(\lambda, \delta, x, y)) \\
\text { as }(x, y, \lambda) & \rightarrow\left(x_{0}, y_{0}, \lambda_{0}\right) .
\end{aligned}
$$

(iii) For every $\xi>0$

$$
\begin{aligned}
& \iint_{\xi \leq \sqrt{s^{2}+t^{2}}} K_{\lambda}\left(\sqrt{s^{2}+t^{2}}\right) d s d t=o(\Delta(\lambda, \delta, x, y)) \\
& \text { as }(x, y, \lambda) \rightarrow\left(x_{0}, y_{0}, \lambda_{0}\right) .
\end{aligned}
$$

Then at each p-generalized Lebesgue point off $\in L_{p}\left(\mathbb{R}^{2}\right)$ we have as $(x, y, \lambda) \rightarrow\left(x_{0}, y_{0}, \lambda_{0}\right)$

$$
\left|L_{\lambda}(f ; x, y)-f\left(x_{0}, y_{0}\right)\right|=o\left(\Delta(\lambda, \delta, x, y)^{\frac{1}{p}}\right) .
$$

Proof In view of the equality of the Lebesgue-Stieltjes integrals we have

$$
\begin{aligned}
& \int_{x_{0}-\delta}^{x_{0}+\delta} \int_{y_{0}-\delta}^{y_{0}+\delta} K_{\lambda}\left(\sqrt{(s-x)^{2}+(t-y)^{2}}\right) d\left[\left(x_{0}-s\right)^{p(\alpha+1)}\left(y_{0}-t\right)^{p(\alpha+1)}\right] \\
& \quad=(p \alpha+p)^{2} \int_{x_{0}-\delta}^{x_{0}+\delta} \int_{y_{0}-\delta}^{y_{0}+\delta}\left(x_{0}-s\right)^{p(\alpha+1)-1}\left(y_{0}-t\right)^{p(\alpha+1)-1} K_{\lambda}\left(\sqrt{(s-x)^{2}+(t-y)^{2}}\right) d s d t .
\end{aligned}
$$


By Theorem 4.1 and Theorem 4.2 we can write, for $\delta>0$,

$$
\begin{aligned}
\left|L_{\lambda}(f ; x, y)-f\left(x_{0}, y_{0}\right)\right|^{p} & 2^{2 p} \int_{\delta \leq \sqrt{s^{2}+t^{2}}}\left|K_{\lambda}\left(\sqrt{t^{2}+s^{2}}\right)\right|\|f\|_{L_{p}\left(\mathbb{R}^{2}\right)}^{p} \\
& \left.+\left|f\left(x_{0}, y_{0}\right)\right|^{p} \iint_{\delta \leq \sqrt{s^{2}+t^{2}}} K_{\lambda}\left(\sqrt{s^{2}+t^{2}}\right) d s d t\right\} \\
& \times\left(\iint_{\mathbb{R}^{2}} K_{\lambda}\left(\sqrt{(s-x)^{2}+(t-y)^{2}}\right) d s d t\right)^{\frac{P}{q}} \\
& +2^{p} \varepsilon^{p}(p \alpha+p)^{2} \int_{x_{0}-\delta}^{x_{0}+\delta} \int_{y_{0}-\delta}^{y_{0}+\delta}\left|x_{0}-s\right|^{p(\alpha+1)-1}\left|y_{0}-t\right|^{p(\alpha+1)-1} \\
& \times K_{\lambda}\left(\sqrt{(s-x)^{2}+(t-y)^{2}}\right) d s d t \\
& \times\left(\iint_{\mathbb{R}^{2}} K_{\lambda}\left(\sqrt{(s-x)^{2}+(t-y)^{2}}\right) d s d t\right)^{\frac{p}{q}} \\
& +2^{p}\left|f\left(x_{0}, y_{0}\right)\right|^{p}\left|\iint_{\mathbb{R}^{2}} K_{\lambda}\left(\sqrt{(s-x)^{2}+(t-y)^{2}}\right) d s d t-1\right|^{p}
\end{aligned}
$$

From (i)-(iii) and using class $A$ conditions we have the desired result:

$$
\left|L_{\lambda}(f ; x, y)-f\left(x_{0}, y_{0}\right)\right|=o\left(\Delta(\lambda, \delta, x, y)^{\frac{1}{p}}\right)
$$

Example 5.1 Let $\Lambda=(0, \infty), \lambda_{0}=0$, and

$$
H_{\lambda}(s, t)=\frac{1}{4 \pi \lambda} e^{\frac{-\left(s^{2}+t^{2}\right)}{4 \lambda}} .
$$

To verify that $K_{\lambda}\left(\sqrt{s^{2}+t^{2}}\right)=\frac{1}{4 \pi \lambda} e^{\frac{-\left(\sqrt{s^{2}+t^{2}}\right)^{2}}{4 \lambda}}$ satisfies the hypotheses of Theorem 4.1 and Theorem 4.2, see [15]. Since $K_{\lambda}\left(\sqrt{s^{2}+t^{2}}\right)$ tends to infinity as $\lambda$ tends to zero at $(s, t)=(0,0)$, condition (b) of class $A$ is satisfied.

Let $p=\frac{3}{2}, \alpha=\frac{1}{3}$, and $\left(x_{0}, y_{0}\right)=(0,0)$. Hence we obtain

$$
\begin{aligned}
\Delta(\lambda, \delta, x, y)= & \int_{-\delta}^{+\delta} \int_{-\delta}^{+\delta}|s||t| \frac{1}{4 \pi \lambda} e^{\frac{-\left((s-x)^{2}+(t-y)^{2}\right)}{4 \lambda}} d s d t \\
= & \frac{1}{2 \pi} e^{\frac{2 \delta^{2}+y^{2}+x^{2}}{4 \lambda}}\left(2 \sqrt{\lambda}\left(e^{\frac{\delta^{2}}{4 \lambda}}-e^{\frac{\delta x}{2 \lambda}}\right)\right. \\
& \left.+e^{\frac{\delta^{2}+x^{2}}{4 \lambda}} \sqrt{\pi} x\left(\operatorname{Erf}\left(\frac{\delta-x}{2 \sqrt{\lambda}}\right)+\operatorname{Erf}\left(\frac{x}{2 \sqrt{\lambda}}\right)\right)\right) \\
& \times\left(2 \sqrt{\lambda}\left(e^{\frac{\delta^{2}}{4 \lambda}}-e^{\frac{\delta y}{2 \lambda}}\right)+e^{\frac{\delta^{2}+y^{2}}{4 \lambda}} \sqrt{\pi} y\left(\operatorname{Erf}\left(\frac{\delta-y}{2 \sqrt{\lambda}}\right)+\operatorname{Erf}\left(\frac{y}{2 \sqrt{\lambda}}\right)\right)\right),
\end{aligned}
$$

where

$$
\operatorname{Erf}(x)=\frac{2}{\sqrt{\pi}} \int_{0}^{x} e^{-t^{2}} d t .
$$


In order to find for which $\delta>0$ condition (i) in Theorem 5.1 is satisfied, let $\Delta(\lambda, \delta, x, y) \rightarrow 0$ as $(x, y, \lambda) \rightarrow(0,0,0)$. Hence

$$
\lim _{(x, y, \lambda) \rightarrow(0,0,0)} \Delta(\lambda, \delta, x, y)=0
$$

if and only if $\delta^{2}=o(\lambda)$. Consequently the following equation holds:

$$
\begin{aligned}
\Delta(\lambda, \delta, x, y) & =\int_{-\delta}^{+\delta} \int_{-\delta}^{+\delta}|s||t| \frac{1}{4 \pi \lambda} e^{\frac{-\left((s-x)^{2}+(t-y)^{2}\right)}{4 \lambda}} d s d t \\
& =2 \int_{[0, \lambda] \times[0, \lambda]}|s||t| \frac{1}{4 \pi \lambda} e^{\frac{-\left((s-x)^{2}+(t-y)^{2}\right)}{4 \lambda}} d s d t .
\end{aligned}
$$

From the above equation we see that

$$
\Delta(\lambda, \delta, x, y)=O(\lambda)
$$

From [15] it is clear that the conditions (ii) and (iii) in Theorem 5.1 are satisfied. Hence

$$
\left|L_{\lambda}(f ; x, y)-f\left(x_{0}, y_{0}\right)\right|=o\left(\Delta(\lambda, \delta, x, y)^{\frac{2}{3}}\right)=o\left(\lambda^{\frac{2}{3}}\right)
$$

\section{Competing interests}

The authors declare that they have no competing interests.

\section{Authors' contributions}

All authors contributed equally to the writing of this paper. All authors read and approved the final manuscript.

\section{Author details}

'Department of Mathematics, Faculty of Arts and Science, Gaziantep University, Gaziantep, Turkey. ${ }^{2}$ Department of Mathematics, Faculty of Science, Karabuk University, Karabuk, Turkey. ${ }^{3}$ Department of Mathematics, Faculty of Science, Ankara University, Tandogan, Ankara, Turkey.

\section{Acknowledgements}

The authors thank the referees for their valuable comments and suggestions for the improvement of the manuscript.

Received: 22 July 2014 Accepted: 27 October 2014 Published: 04 Nov 2014

\section{References}

1. Taberski, R: Singular integrals depending on two parameters. Ann. Pol. Math. 15, 97-115 (1962)

2. Gadjiev, AD: The order of convergence of singular integrals which depend on two parameters. In: Special Problems of Functional Analysis and Their Applications to the Theory of Differential Equations and the Theory of Functions, pp. 40-44. Izdat. Akad. Nauk Azerbaĭdažan. SSR., Baku (1968)

3. Rydzewska, B: Approximation des fonctions par des intégrales singulières ordinaires. Fasc. Math. 7, 71-81 (1973)

4. Karsli, H, Ibikli, I: Approximation properties of convolution type singular integral operators depending on two parameters and of their derivatives in $L_{1}(a, b)$. In: Proc. 16th Int. Conf. Jangjeon Math. Soc., vol. 16, pp. 66-76 (2005)

5. Karsli, $\mathrm{H}$, Ibikli, E: On convergence of convolution type singular integral operators depending on two parameters. Fasc. Math. 38, 25-39 (2007)

6. Karsli, $\mathrm{H}$ : Convergence and rate of convergence by nonlinear singular integral operators depending on two parameters. Appl. Anal. 85(6-7), 781-791 (2006)

7. Karsli, $\mathrm{H}$ : On the approximation properties of a class of convolution type nonlinear singular integral operators. Georgian Math. J. 15(1), 77-86 (2008)

8. Bardaro, C, Gori Cocchieri, C: On the degree of approximation for a class of singular integrals. Rend. Mat. (7) 4(4), 481-490 (1984) (Italian)

9. Bardaro, C: On approximation properties for some classes of linear operators of convolution type. Atti Semin. Mat. Fis. Univ. Modena 33(2), 329-356 (1984)

10. Bardaro, C, Mantellini, I: Pointwise convergence theorems for nonlinear Mellin convolution operators. Int. J. Pure Appl. Math. 27(4), 431-447 (2006)

11. Bardaro, C, Karsli, H, Vinti, G: On pointwise convergence of linear integral operators with homogeneous kernel. Integral Transforms Spec. Funct. 19(6), 429-439 (2008) 
12. Bardaro, C, Vinti, G, Karsli, H: Nonlinear integral operators with homogeneous kernels: pointwise approximation theorems. Appl. Anal. 90(3-4), 463-474 (2011)

13. Bardaro, C, Karsli, H, Vinti, G: On pointwise convergence of Mellin type nonlinear $m$-singular integral operators. Commun. Appl. Nonlinear Anal. 20(2), 25-39 (2013)

14. Taberski, R: On double integrals and Fourier series. Ann. Pol. Math. 15, 97-115 (1964)

15. Siudut, S: On the convergence of double singular integrals. Comment. Math. Prace Mat. 28(1), 143-146 (1988)

16. Siudut, S: A theorem of Romanovski type for double singular integrals. Comment. Math. Prace Mat. 29, 277-289 (1989)

17. Yilmaz, MM, Serenbay, SK, Ibikli, E: On singular integrals depending on three parameters. Appl. Math. Comput. 218(3), 1132-1135 (2011)

18. Serenbay, SK, Dalmanoglu, O, Ibikli, E: On convergence of singular integral operators with radial kernels. In: Approximation Theory XIV: San Antonio 2013. Springer Proceedings in Mathematics and Statistics, vol. 83, pp. 295-308 (2014)

19. Ghorpade, SR, Limaye, BV: A Course in Multivariable Calculus and Analysis. Springer, New York (2010)

20. Bochner, S, Chandrasekharan, K: Fourier Transforms. Annals of Mathematics Studies, vol. 19. Princeton University Press, Princeton (1949)

21. Yilmaz, MM: On convergence of singular integral operators depending on three parameters with radial kernels. Int. J. Math. Anal. 4(39), 1923-1928 (2010)

10.1186/1687-1847-2014-287

Cite this article as: Yilmaz et al.: A note on rate of convergence of double singular integral operators. Advances in Difference Equations 2014, 2014:287

\section{Submit your manuscript to a SpringerOpen ${ }^{\circ}$ journal and benefit from:}

- Convenient online submission

- Rigorous peer review

- Immediate publication on acceptance

- Open access: articles freely available online

- High visibility within the field

- Retaining the copyright to your article 